\title{
A METHOD WITH FEEDBACK FOR AGGREGATION OF GROUP INCOMPLETE PAIR-WISE COMPARISONS USING SCALES WITH DIFFERENT NUMBERS OF GRADES
}

\begin{abstract}
A method for aggregation of expert estimates in small groups is proposed. It allows to derive priority vector based on group incomplete pair-wise comparisons and to organize feedback with experts in order to achieve agreement. Every expert is given an opportunity to use the scale, in which the degree of detail (number of points/grades) most adequately reflects this expert's competence in the issue under consideration, for every single pair comparison.

Keywords: group decision making, incomplete pair-wise comparisons, combinatorial method, feedback with experts, scales with different numbers of grades.
\end{abstract}

\section{Introduction}

To improve the reliability of the information obtained from experts in the AHP/ANP it is desirable to use a group of experts (Saaty \& Peniwati, 2007). Agreement (consistency, compatibility) of individual expert estimates is an important aspect of group decisionmaking. In order to achieve the level of consistency of individual pair-wise comparisons, provided by experts, which would be sufficient for aggregation of these pair-wise comparisons, it is necessary to organise feedback with experts. Well-known approaches to aggregation of individual expert estimates are described by (Forman \& Peniwati, 1998). The disadvantage of these methods is that they do not allow to organise feedback with experts in order to improve agreement. A new one-phase aggregation method, using combinatorial enumeration of spanning trees, obtained from individual pair-wise comparison matrices, with feedback, is proposed. Another advantage of the method (beside feedback) is that it allows us to use scales with different numbers of grades for every single pair-wise comparison.

\section{Relevance}

The benefits of Combinatorial method for aggregation effectiveness were shown in (Tsyganok, 2010). Beside that, effectiveness of usage of scales with different numbers of grades for each new comparison (as the competence (knowledge level) of experts is different for each specific issue) was experimentally confirmed (Tsyganok, Kadenko \& Andriychuk, 2015). As the aggregation of expert estimates is, surely, possible when the sufficient level of their agreement is reached, it can be stated that the development of a Method with Feedback for Aggregation of Group Incomplete Pair-Wise Comparisons using Scales with Different Numbers of Grades is relevant.

\section{Problem statement}

The formal statement of alternative weight calculation problem in our case can be as follows. What is given: $A_{l}, l \in[1 . . m]-$ expert pair-wise comparison matrices (PCM) with dimensionality of $n \times n$, which have the following properties: 1) matrices are reciprocallysymmetrical; 2) matrices are multiplicative; 3) in the general case, matrices are incomplete; 4) every single element of a PCM is obtained in some scale, which is assigned a weight coefficient $\left.s_{h}, h \in[0 . .8]\right) ; c_{l}, l \in[1 . . m]$ - relative competence of experts in the group $\left(\Sigma_{c_{l}}=1\right)$. We should find: The resulting object weight (priority) vector $w_{k}$, $k \in[1 . . n]\left(\sum w_{k}=1\right)$.

\section{Stages of problem solution}

1) Bringing the estimates given by different experts in different scales to a unified scale. 
A Method with Feedback for Aggregation of Group Incomplete Pair-Wise Comparisons using Scales with Different Numbers of Grades (ISAHP-2016, London, U.K.)

2) Weighting of estimates. An estimate provided in the more informative scale should "weight" more than the estimate, provided by an expert using the less detailed scale. The weight should be proportional to the quantity of information (according to Hartley's formula: $I=\log _{2} N$, where $N$ is the number of expert estimation scale grades).

3) Ensuring completeness and agreement of the estimates. In order to fully utilize the redundancy of expert information and to reach high efficiency, it is suggested to aggregate individual PCMs using the so-called combinatorial method (Tsyganok, 2010). Before aggregation of estimates, completeness and agreement of individual PCMs should be checked. Completeness and agreement are ensured as follows. In combinatorial method priority vectors are calculated based on some basic sets of pair-wise comparisons. If such basic set is not complete, the priority vector cannot be calculated, so, in such case, the expert should be re-addressed with a request to provide the missing basic pair-wise comparisons, and, thus, ensure completeness of the set. If the matrices are not compatible enough, the results of the whole expert examination, even if they can be obtained, will be less credible. For agreement check (and, if necessary, improvement) the Double Entropy Agreement Index (Olenko \& Tsyganok, 2016) is suggested for use. In terms of agreement and its improvement, the combinatorial method has the aforementioned advantages over group aggregation methods, described by (Forman \& Peniwati, 1998). An ideally consistent aggregate PCM is built as described in (Tsyganok 2010). If agreement improvement is required, pair-wise comparisons from these matrices are adjusted in such a way that they become closer to the respective elements of the ideally consistent aggregate matrix, so that agreement of PCMs altogether are improved.

4) Aggregation of estimates. After the agreement improvement is done, alternative weights can be calculated based on the corrected PCMs as described in (Tsyganok 2010).

\section{Conclusions}

A Method with Feedback for Aggregation of Group Incomplete Pair-Wise Comparisons using Scales with Different Numbers of Grades is suggested. The method allows the expert group to reach consensus, which is necessary for aggregation of individual estimates. The opportunity to use scales with different numbers of grades increases the credibility of expert information. The method allows an expert to abstain from making a comparison, if (s)he doesn't feel competent enough. In such case the PCM is incomplete, and the number of basic pair-wise comparison sets (spanning trees), by which the aggregate matrix is calculated, decreases.

\section{Key References}

Forman, E. \& Peniwati, K. (1998). Aggregating individual judgments and priorities with the analytic hierarchy process. European Journal of Operational Research, 108(1), 131145.

Olenko, A. \& Tsyganok, V. (2016). Double Entropy Inter-Rater Agreement Indices. Applied Psychological Measurement, 40(1), 37-55.

Saaty, T.L., \& Peniwati, K. (2007). Group decision-making: Drawing out and reconciling differences. Pittsburgh, PA: RWS Publications.

Tsyganok, V.V. (2010). Investigation of the aggregation effectiveness of expert estimates obtained by the pairwise comparison method. Mathematical and Computer Modelling, 52(3-4), 538-544.

Tsyganok, V.V., Kadenko, S.V., \& Andiichuk O.V. (2015). Using different pair-wise comparison scales for developing industrial strategies. International Journal of Management and Decision Making, 14(3), 224-250. 